https://doi.org/10.15407/ujpe63.4.350

A.S. VDOVYCH, ${ }^{1}$ I.R. ZACHEK ${ }^{2}$ R.R. LEVITSKII ${ }^{1}$

${ }^{1}$ Institute for Condensed Matter Physics, Nat. Acad. Sci. of Ukraine

(1, Svientsitskii Str., Lviv 79011, Ukraine; e-mail: vas@icmp.lviv.ua)

${ }^{2}$ Lviv Polytechnic National University

(12, Bandera Str., Lviv 79013, Ukraine)

\title{
INFLUENCE OF LONGITUDINAL ELECTRIC FIELD ON THERMODYNAMIC PROPERTIES OF $\mathrm{NH}_{3} \mathrm{CH}_{2} \mathrm{COOH} \cdot \mathrm{H}_{2} \mathrm{PO}_{3}$ FERROELECTRIC
}

\begin{abstract}
Using a modified model of ferroelectric glycine phosphite by considering the piezoelectric coupling with strains $\varepsilon_{i}$ within the two-particle cluster approximation, the expressions for the polarization vector, static dielectric permittivity tensor, piezoelectric coefficients, and elastic constants of the crystal in the presence of a longitudinal electric field $E_{2}$ are calculated. An analysis of the influence of this field on thermodynamic characteristics of the crystal is carried out. The dependence of effective dipole moments on order parameters is taken into account. This allowed us to agree the effective dipole moments in the ferro- and paraelectric phases and to describe the smearing of phase transition under the influence of an electric field. The satisfactory quantitative description of the available experimental data for these characteristics has been obtained at the proper choice of the model parameters.

Keywords: ferroelectrics, electric field, polarization, dielectric permittivity, piezoelectric coefficients.
\end{abstract}

\section{Introduction}

One of the actual problems in physics of ferroelectric materials is the study of the effects that appear under the action of an external electric field. It can be a powerful tool for the purposeful control of their physical characteristics. The effects of the action of external fields depend both on the intensity and the type of such an action, and on the properties of the materials. The application of an electric field is a very important instrument for the investigation of ferroelectric materials with a complex spatial arrangement of the local effective dipole moments. Consequently, phase transitions with different order parameters connected with one another can take place in these materials. In particular, it appears possible to influence this system by means of longitudinal and transverse electric fields.

Crystal $\mathrm{NH}_{3} \mathrm{CH}_{2} \mathrm{COOH} \cdot \mathrm{H}_{2} \mathrm{PO}_{3}$ (glycinium phosphite - GPI) belongs to the family of ferroelectrics with hydrogen bonds $[1,2]$. At room temperature, this crystal is in the paraelectric phase and has a monoclinic structure (space group P2 1 /a) [3]. But, at the temperature about $225 \mathrm{~K}$, the crystal passes to

(C) A.S. VDOVYCH, I.R. ZACHEK, R.R. LEVITSKII, 2018 350 the ferroelectric phase (space group $\mathrm{P} 2{ }_{1}$ ). It is one of the most interesting examples of a crystal sensitive to the influence of both longitudinal and transverse electric fields. In particular, there was experimentally ascertained in $[4,5]$ that its transverse dielectric permittivity $\varepsilon_{z z}$ in the ferroelectric phase significantly increases under the influence of a transverse field $E_{z}$.

In $[4,6]$, a microscopic approach was proposed for the theoretical study of dielectric properties of GPI crystals, on the basis of which the transverse field effect on the permittivity $\varepsilon_{z z}$ was explained qualitatively properly.

In [7], this approach was supplemented by allowance for the piezoelectric coupling of the proton subsystem with the lattice strain. This allowed one to calculate the expressions for the spontaneous polarization vector, static dielectric permittivity tensor, piezoelectric coefficients, elastic constants, and molar heat capacity of the crystal and to obtain the good quantitative description of the experimental data for these characteristics. Moreover, on the basis of a modified model of GPI, we quantitatively properly described the influence of transverse fields $E_{x}$ and $E_{z}[8]$, hydrostatic [9] and uniaxial [10] pressures

ISSN 2071-0194. Ukr. J. Phys. 2018. Vol. 63, No. 4 
on the phase transition and thermodynamic characteristics of GPI crystal.

Experimental data [11] show that, in the presence of a longitudinal field $E_{y}$, the phase transition smears, and the temperature dependence of the longitudinal permittivity $\varepsilon_{y y}(T)$ has rounded maximum. At the same time, in the model proposed in [7], the effective dipole moments with different values of longitudinal components in the para- and ferroelectric phases are used for the description of the longitudinal dielectric permittivity $\varepsilon_{y y}$. This leads to the appearance of a jump on the curve $\varepsilon_{y y}(T)$ instead of a smoothed maximum in the presence of an external field $E_{y}$. Therefore, in the present paper for the proper description of the phase transition smearing, we modify the model [7], by supposing that the effective dipole moment on the hydrogen bond depends on the order parameter on this bond, because this parameter continuously depends on the temperature near the phase transition point.

\section{Thermodynamic Characteristics}

In the model proposed in [7], we consider the system of protons in GPI localized on $\mathrm{O}-\mathrm{H}$...O bonds, which form chains along the $c$-axis of the crystal.

Dipole moments $\mathbf{d}_{q f}(f=1, \ldots, 4)$ are ascribed to the protons on the bonds. In the ferroelectric phase, the dipole moments compensate one another $\left(\mathbf{d}_{q 1}\right.$ with $\mathbf{d}_{q 3}, \mathbf{d}_{q 2}$ with $\left.\mathbf{d}_{q 4}\right)$ in directions $Z$ and $X$ $(X \perp(b, c), Y\|b, Z\| c)$, and simultaneously supplement one another in direction $Y$, creating a spontaneous polarization. Vectors $\mathbf{d}_{q f}$ are oriented at some angles to crystalographic axes and have longitudinal and transverse components along $b$-axis (Fig. 1). For the better understanding of the model, only phosphite groups are shown in the figure.

The pseudospin variables $\frac{\sigma_{q 1}}{2}, \ldots, \frac{\sigma_{q 4}}{2}$ describe reorientation of the dipole moments of the base units: $\mathbf{d}_{q f}=\boldsymbol{\mu}_{f} \frac{\sigma_{q f}}{2}$. The mean values $\left\langle\frac{\sigma}{2}\right\rangle=\frac{1}{2}\left(n_{a}-n_{6}\right)$ are connected with differences in the occupancies of the two possible molecular positions, $n_{a}$ and $n_{b}$.

Below, for the components of vectors and tensors, we use the notations 1,2 , and 3 instead of $x, y$, and $z$ for convenience. The Hamiltonian of the proton subsystem of GPI, which involves the short-range and long-range interactions and the applied electric fields $E_{1}, E_{2}$, and $E_{3}$ along the positive directions of the Cartesian axes $X, Y$ and $Z$ can be written
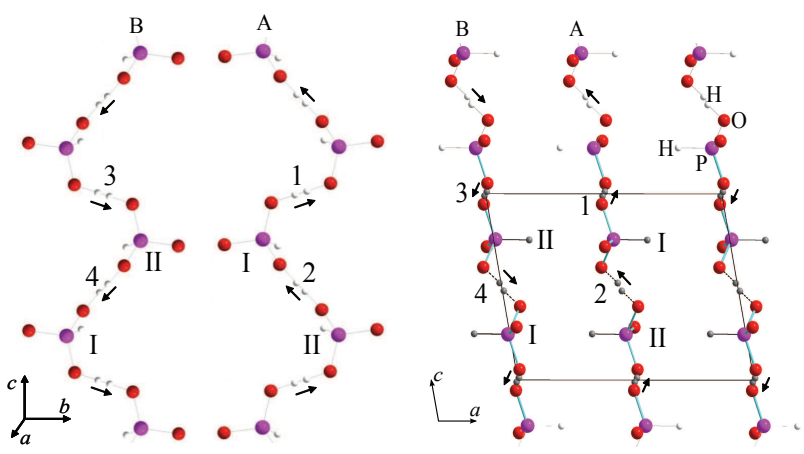

Fig. 1. Orientations of vectors $\mathbf{d}_{q f}$ in the primitive cell in the ferroelectric phase [7]

in such a way:

$\hat{H}=N U_{\text {seed }}+\hat{H}_{\text {short }}+\hat{H}_{\text {long }}+\hat{H}_{E}+\hat{H}_{E}^{\prime}$,

where $N$ is the total number of primitive cells. The first term in (2.1) is the "seed" energy, which relates to the heavy ion sublattice and does not depend explicitly on the configuration of the proton subsystem. It includes the elastic, piezolectric, and dielectric parts expressed in terms of the electric fields $E_{i}$ and strains $\varepsilon_{i}$ as follows:

$U_{\text {seed }}=v\left(\frac{1}{2} \sum_{i, i^{\prime}=1}^{3} c_{i i^{\prime}}^{E 0}(T) \varepsilon_{i} \varepsilon_{i^{\prime}}+\frac{1}{2} \sum_{j=4}^{6} c_{j j}^{E 0}(T) \varepsilon_{j}^{2}+\right.$

$+\sum_{i=1}^{3} c_{i 5}^{E 0}(T) \varepsilon_{i} \varepsilon_{5}+c_{46}^{E 0}(T) \varepsilon_{4} \varepsilon_{6}-$

$-\sum_{i=1}^{3} e_{2 i}^{0} \varepsilon_{i} E_{2}-e_{25}^{0} \varepsilon_{5} E_{2}-e_{14}^{0} \varepsilon_{4} E_{1}-$

$-e_{16}^{0} \varepsilon_{6} E_{1}-e_{34}^{0} \varepsilon_{4} E_{3}-e_{36}^{0} \varepsilon_{6} E_{3}-$

$\left.-\frac{1}{2} \chi_{11}^{\varepsilon 0} E_{1}^{2}-\frac{1}{2} \chi_{22}^{\varepsilon 0} E_{2}^{2}-\frac{1}{2} \chi_{33}^{\varepsilon 0} E_{3}^{2}-\chi_{31}^{\varepsilon 0} E_{3} E_{1}\right)$.

The parameters $c_{i j}^{E 0}(T), e_{i j}^{0}$, and $\chi_{i j}^{\varepsilon 0}$ are the so-called "seed" elastic constants, piezoelectric stresses, and dielectric susceptibilities, respectively, and $v$ is the volume of a primitive cell.

Other terms in (2.1) describe the pseudospin part of the Hamiltonian. In particular, the second term in (2.1) is the Hamiltonian of short-range interactions:

$\hat{H}_{\text {short }}=2 w \sum_{q q^{\prime}}\left(\frac{\sigma_{q 1}}{2} \frac{\sigma_{q 2}}{2}+\frac{\sigma_{q 3}}{2} \frac{\sigma_{q 4}}{2}\right) \times$
$\times\left(\delta_{\mathbf{R}_{q} \mathbf{R}_{q^{\prime}}}+\delta_{\mathbf{R}_{q}+\mathbf{R}_{\mathbf{c}}, \mathbf{R}_{q^{\prime}}}\right)$. 
In $(2.3), \sigma_{q f}$ is the $z$-component of the pseudospin operator that describes the state of the $f$-th bond $(f=1,2,3,4)$ in the $q$-th cell. The first Kronecker delta corresponds to the interaction between protons in the chains near the tetrahedra $\mathrm{HPO}_{3}$ of type "I" (Fig. 1), where the second one near the tetrahedra $\mathrm{HPO}_{3}$ of type "II", $\mathbf{R}_{\mathbf{c}}$ is the lattice vector along the $O Z$-axis. Contributions into the energy of interactions between protons near tetrahedra of different types, as well as the mean values of the pseudospins $\eta_{f}=\left\langle\sigma_{q f}\right\rangle$, which are related to tetrahedra of different types, are equal. The parameter $w$, which describes the short-range interactions within chains, is expanded linearly into series in strains $\varepsilon_{i}$ :

$w=w^{0}+\sum_{i=1}^{6} \delta_{i} \varepsilon_{i}$

The third term in (2.1) describes the long-range dipole-dipole interactions and indirect (through the lattice vibrations) interactions between protons, which are taken into account in the mean field approximation:

$$
\begin{aligned}
& \hat{H}_{\text {long }}=\frac{1}{2} \sum_{\substack{q q^{\prime} \\
f f^{\prime}}} J_{f f^{\prime}}\left(q q^{\prime}\right) \frac{\left\langle\sigma_{q f}\right\rangle}{2} \frac{\left\langle\sigma_{q^{\prime} f^{\prime}}\right\rangle}{2}- \\
& -\sum_{\substack{q q^{\prime} \\
f f^{\prime}}} J_{f f^{\prime}}\left(q q^{\prime}\right) \frac{\left\langle\sigma_{q^{\prime} f^{\prime}}\right\rangle}{2} \frac{\sigma_{q f}}{2} .
\end{aligned}
$$

The Fourier transforms of interaction constants $J_{f f^{\prime}}=\sum_{q^{\prime}} J_{f f^{\prime}}\left(q q^{\prime}\right)$ at $\mathbf{k}=0$ are linearly expanded in the strains $\varepsilon_{i}$ :

$J_{f f^{\prime}}=J_{f f^{\prime}}^{0}+\frac{\partial J_{f f^{\prime}}}{\partial \varepsilon_{i}} \varepsilon_{i}=J_{f f^{\prime}}^{0}+\sum_{i=1}^{6} \psi_{f f^{\prime} i} \varepsilon_{i}$.

As a result, (2.5) can be written as

$\hat{H}_{\text {long }}=N H^{0}+\hat{H}_{s}$,

where

$$
\begin{aligned}
& H^{0}=\frac{1}{8} J_{11}\left(\eta_{1}^{2}+\eta_{3}^{2}\right)+\frac{1}{8} J_{22}\left(\eta_{2}^{2}+\eta_{4}^{2}\right)+ \\
& +\frac{1}{4} J_{13} \eta_{1} \eta_{3}+\frac{1}{4} J_{24} \eta_{2} \eta_{4}+ \\
& +\frac{1}{4} J_{12}\left(\eta_{1} \eta_{2}+\eta_{3} \eta_{4}\right)+\frac{1}{4} J_{14}\left(\eta_{1} \eta_{4}+\eta_{2} \eta_{3}\right), \\
& \hat{H}_{s}=\sum_{q}\left(\mathcal{H}_{1} \frac{\sigma_{q 1}}{2}+\mathcal{H}_{2} \frac{\sigma_{q 2}}{2}+\mathcal{H}_{3} \frac{\sigma_{q 3}}{2}+\mathcal{H}_{4} \frac{\sigma_{q 4}}{2}\right) .
\end{aligned}
$$

In (2.9), the following notations are used:

$$
\begin{aligned}
\mathcal{H}_{1} & =\frac{1}{2} J_{11} \eta_{1}+\frac{1}{2} J_{12} \eta_{2}+\frac{1}{2} J_{13} \eta_{3}+\frac{1}{2} J_{14} \eta_{4}, \\
\mathcal{H}_{2} & =\frac{1}{2} J_{22} \eta_{2}+\frac{1}{2} J_{12} \eta_{1}+\frac{1}{2} J_{24} \eta_{4}+\frac{1}{2} J_{14} \eta_{3}, \\
\mathcal{H}_{3} & =\frac{1}{2} J_{11} \eta_{3}+\frac{1}{2} J_{12} \eta_{4}+\frac{1}{2} J_{13} \eta_{1}+\frac{1}{2} J_{14} \eta_{2}, \\
\mathcal{H}_{4} & =\frac{1}{2} J_{22} \eta_{4}+\frac{1}{2} J_{12} \eta_{3}+\frac{1}{2} J_{24} \eta_{2}+\frac{1}{2} J_{14} \eta_{1} .
\end{aligned}
$$

The fourth term in (2.1) describes the interactions of pseudospins with an external electric field:

$\hat{H}_{E}=-\sum_{q f} \boldsymbol{\mu}_{f} \mathbf{E} \frac{\sigma_{q f}}{2}$.

Here, $\boldsymbol{\mu}_{1}=\left(\mu_{13}^{x}, \mu_{13}^{y}, \mu_{13}^{z}\right), \boldsymbol{\mu}_{3}=\left(-\mu_{13}^{x}, \mu_{13}^{y},-\mu_{13}^{z}\right)$, $\boldsymbol{\mu}_{2}=\left(-\mu_{24}^{x},-\mu_{24}^{y}, \mu_{24}^{z}\right), \boldsymbol{\mu}_{4}=\left(\mu_{24}^{x},-\mu_{24}^{y},-\mu_{24}^{z}\right)$ are the effective dipole moments per one pseudospin.

The first four terms in Hamiltonian (2.1) describe the model [7]. The term $\hat{H}_{E}^{\prime}$ in hamiltonian (2.1) is introduced in the present model for the first time. It accounts for the above-mentioned dependence of longitudinal components of dipole moments on the mean value of pseudospin $s_{f}$ :

$$
\begin{aligned}
& \hat{H}_{E}^{\prime}=-\sum_{q f} s_{f}^{2} \mu_{f}^{\prime} E_{2} \frac{\sigma_{q f}}{2}= \\
& =-\sum_{q f}\left(\frac{1}{N} \sum_{q^{\prime}} \sigma_{q^{\prime} f}\right)^{2} \mu_{f}^{\prime} E_{2} \frac{\sigma_{q f}}{2} .
\end{aligned}
$$

Here, we use corrections to dipole moments $s_{f}^{2} \mu_{f}^{\prime}$ instead of $s_{f} \mu_{f}^{\prime}$ because of the symmetry considerations: the energy should not be changed, when the field and all pseudospins change their sign.

The term $\hat{H}_{E}^{\prime}$, as well as long-range interactions, is taken into account in the mean field approximation:

$$
\begin{aligned}
& \hat{H}_{E}^{\prime}=-\sum_{q f}\left(\frac{1}{N} \sum_{q^{\prime}} \sigma_{q^{\prime} f}\right)^{2} \mu_{f}^{\prime} E_{2} \frac{\sigma_{q f}}{2}= \\
& =-\frac{1}{N^{2}} \sum_{q f} \sum_{q^{\prime}} \sum_{q^{\prime \prime}} \sigma_{q f} \sigma_{q^{\prime} f} \sigma_{q^{\prime \prime} f} \frac{\mu_{f}^{\prime} E_{2}}{2} \approx \\
& \approx-\frac{1}{N^{2}} \sum_{q f} \sum_{q^{\prime}} \sum_{q^{\prime \prime}}\left(\left(\sigma_{q f}+\sigma_{q^{\prime} f}+\sigma_{q^{\prime \prime} f}\right) \eta_{f}^{2}-2 \eta_{f}^{3}\right) \frac{\mu_{f}^{\prime} E_{2}}{2}= \\
& =-\sum_{q} \sum_{f=1}^{4}\left(3 \sigma_{q f} \eta_{f}^{2}-2 \eta_{f}^{3}\right) \frac{\mu_{f}^{\prime} E_{2}}{2}=
\end{aligned}
$$

ISSN 2071-0194. Ukr. J. Phys. 2018. Vol. 63, No. 4 
$=-3 \sum_{q} \sum_{f=1}^{4} \frac{\sigma_{q f}}{2} \eta_{f}^{2} \mu_{f}^{\prime} E_{2}+N \sum_{f=1}^{4} \eta_{f}^{3} \mu_{f}^{\prime} E_{2}$.

The two-particle cluster approximation for shortrange interactions is used in the calculation of thermodynamic characteristics of GPI. In this approximation, the thermodynamic potential is given by

$G=N U_{\text {seed }}+N H^{0}+N \sum_{f=1}^{4} \eta_{f}^{3} \mu_{f}^{\prime} E_{2}-N v \sum_{i=1}^{6} \sigma_{i} \varepsilon_{i}-$

$-k_{\mathrm{B}} T \sum_{q}\left\{2 \ln \mathrm{Sp} e^{-\beta \hat{H}_{q}^{(2)}}-\sum_{f=1}^{4} \ln \mathrm{Sp} e^{-\beta \hat{H}_{q f}^{(1)}}\right\}$.

Here, $\hat{H}_{q}^{(2)}, \hat{H}_{q f}^{(1)}$ are two-particle and one-particle Hamiltonians:

$\hat{H}_{q}^{(2)}=-2 w\left(\frac{\sigma_{q 1}}{2} \frac{\sigma_{q 2}}{2}+\frac{\sigma_{q 3}}{2} \frac{\sigma_{q 4}}{2}\right)-$

$-\frac{y_{1}}{\beta} \frac{\sigma_{q 1}}{2}-\frac{y_{2}}{\beta} \frac{\sigma_{q 2}}{2}-\frac{y_{3}}{\beta} \frac{\sigma_{q 3}}{2}-\frac{y_{4}}{\beta} \frac{\sigma_{q 4}}{2}$,

$\hat{H}_{q f}^{(1)}=-\frac{\bar{y}_{f}}{\beta} \frac{\sigma_{q f}}{2}$,

where such notations are used:

$y_{f}=\beta\left(\Delta_{f}+\mathcal{H}_{f}+\boldsymbol{\mu}_{f} \mathbf{E}+3 \eta_{f}^{2} \mu_{f}^{\prime} E_{2}\right)$,

$\bar{y}_{f}=\beta \Delta_{f}+y_{f}$.

The symbols $\Delta_{f}$ are the effective fields created by the neighboring bonds from outside of the cluster. In the cluster approximation, the fields $\Delta_{f}$ can be determined from the self-consistency condition, which states that the mean values of the pseudospins $\left\langle\sigma_{q f}\right\rangle$ calculated with the two-particle and one-particle Gibbs distributions, respectively, should coincide. That is,

$\frac{\operatorname{Sp} \sigma_{q f} e^{-\beta \hat{H}_{q}^{(2)}}}{\operatorname{Sp} e^{-\beta \hat{H}_{q}^{(2)}}}=\frac{\operatorname{Sp} \sigma_{q f} e^{-\beta \hat{H}_{q f}^{(1)}}}{\operatorname{Sp} e^{-\beta \hat{H}_{q f}^{(1)}}}$.

Hence, on the basis of (2.19) with regard for (2.15) and (2.16), we obtain

$\eta_{1,3}=\frac{1}{D}\left[\operatorname{sh} n_{1} \pm \operatorname{sh} n_{2}+a^{2} \operatorname{sh} n_{3} \pm a^{2} \operatorname{sh} n_{4}+\right.$

$\left.+a \operatorname{sh} n_{5}+a \operatorname{sh} n_{6} \mp a \operatorname{sh} n_{7} \pm a \operatorname{sh} n_{8}\right]=\operatorname{th} \frac{\bar{y}_{1,3}}{2}$,

$\eta_{2,4}=\frac{1}{D}\left[\operatorname{sh} n_{1} \pm \operatorname{sh} n_{2}-a^{2} \operatorname{sh} n_{3} \mp a^{2} \operatorname{sh} n_{4} \mp\right.$

$\left.\mp a \operatorname{sh} n_{5} \pm a \operatorname{sh} n_{6}+a \operatorname{sh} n_{7}+a \operatorname{sh} n_{8}\right]=\operatorname{th} \frac{\bar{y}_{2,4}}{2}$,

ISSN 2071-0194. Ukr. J. Phys. 2018. Vol. 63, No. 4 where

$D=\operatorname{ch} n_{1}+\operatorname{ch} n_{2}+a^{2} \operatorname{ch} n_{3}+a^{2} \operatorname{ch} n_{4}+$

$+a \operatorname{ch} n_{5}+a \operatorname{ch} n_{6}+a \operatorname{ch} n_{7}+a \operatorname{ch} n_{8}$,

$a=e^{-\frac{1}{k_{\mathrm{B}} T}\left(w^{0}+\sum_{i=1}^{3} \delta_{i} \varepsilon_{i}+\sum_{j=4}^{6} \delta_{j} \varepsilon_{j}\right)}$,

$n_{1}=\frac{1}{2}\left(y_{1}+y_{2}+y_{3}+y_{4}\right), n_{2}=\frac{1}{2}\left(y_{1}+y_{2}-y_{3}-y_{4}\right)$,

$n_{3}=\frac{1}{2}\left(y_{1}-y_{2}+y_{3}-y_{4}\right), n_{4}=\frac{1}{2}\left(y_{1}-y_{2}-y_{3}+y_{4}\right)$,

$n_{5}=\frac{1}{2}\left(y_{1}-y_{2}+y_{3}+y_{4}\right), n_{6}=\frac{1}{2}\left(y_{1}+y_{2}+y_{3}-y_{4}\right)$,

$n_{7}=\frac{1}{2}\left(-y_{1}+y_{2}+y_{3}+y_{4}\right), n_{8}=\frac{1}{2}\left(y_{1}+y_{2}-y_{3}+y_{4}\right)$.

Taking (2.20) into consideration, we exclude the parameters $\Delta_{f}$ and write $(2.17)$ in such a form:

$y_{1}=\frac{1}{2} \ln \frac{1+\eta_{1}}{1-\eta_{1}}+\beta \nu_{11} \eta_{1}+\beta \nu_{12} \eta_{2}+\beta \nu_{13} \eta_{3}+$

$+\beta \nu_{14} \eta_{4}+\frac{\beta}{2}\left(\boldsymbol{\mu}_{1} \mathbf{E}+3 \eta_{1}^{2} \mu_{1}^{\prime} E_{2}\right)$,

$y_{2}=\beta \nu_{12} \eta_{1}+\frac{1}{2} \ln \frac{1+\eta_{2}}{1-\eta_{2}}+\beta \nu_{22} \eta_{2}+\beta \nu_{14} \eta_{3}+$

$+\beta \nu_{24} \eta_{4}+\frac{\beta}{2}\left(\boldsymbol{\mu}_{2} \mathbf{E}+3 \eta_{2}^{2} \mu_{2}^{\prime} E_{2}\right)$,

$y_{3}=\beta \nu_{13} \eta_{1}+\beta \nu_{14} \eta_{2}+\frac{1}{2} \ln \frac{1+\eta_{3}}{1-\eta_{3}}+\beta \nu_{11} \eta_{3}+$

$+\beta \nu_{12} \eta_{4}+\frac{\beta}{2}\left(\boldsymbol{\mu}_{3} \mathbf{E}+3 \eta_{3}^{2} \mu_{3}^{\prime} E_{2}\right)$,

$y_{4}=\beta \nu_{14} \eta_{1}+\beta \nu_{24} \eta_{2}+\beta \nu_{12} \eta_{3}+$

$+\frac{1}{2} \ln \frac{1+\eta_{4}}{1-\eta_{4}}+\beta \nu_{22} \eta_{4}+\frac{\beta}{2}\left(\boldsymbol{\mu}_{4} \mathbf{E}+3 \eta_{4}^{2} \mu_{4}^{\prime} E_{2}\right)$,

where $\nu_{f f^{\prime}}=\frac{J_{f f^{\prime}}}{4}$.

In the paraelectric and ferroelectric phases in the applied electric field $E_{2}$, the order parameters $\eta_{1}=$ $=\eta_{3}=\eta_{13}, \eta_{2}=\eta_{4}=\eta_{24}$. As a result, Eqs. (2.20) are given by

$$
\begin{aligned}
& \eta_{13}=\frac{1}{\widetilde{D}}\left[\operatorname{sh}\left(y_{13}+y_{24}\right)+a^{2} \operatorname{sh}\left(y_{13}-y_{24}\right)+2 a \operatorname{sh} y_{13}\right], \\
& \eta_{24}=\frac{1}{\widetilde{D}}\left[\operatorname{sh}\left(y_{13}+y_{24}\right)-a^{2} \operatorname{sh}\left(y_{13}-y_{24}\right)+2 a \operatorname{sh} y_{24}\right],
\end{aligned}
$$


where we used the notations

$\tilde{D}=\operatorname{ch}\left(y_{13}+y_{24}\right)+a^{2} \operatorname{ch}\left(y_{13}-y_{24}\right)+$

$+2 a \operatorname{ch} y_{13}+2 a \operatorname{ch} y_{24}+a^{2}+1$,

$y_{13}=\frac{1}{2} \ln \frac{1+\eta_{13}}{1-\eta_{13}}+\beta \nu_{1}^{+} \eta_{13}+\beta \nu_{2}^{+} \eta_{24}+$

$+\frac{\beta}{2}\left(\mu_{13}^{y} E_{2}+3 \eta_{1}^{2} \mu_{13}^{\prime} E_{2}\right)$

$y_{24}=\beta \nu_{2}^{+} \eta_{13}+\frac{1}{2} \ln \frac{1+\eta_{24}}{1-\eta_{24}}+\beta \nu_{3}^{+} \eta_{24}-$

$-\frac{\beta}{2}\left(\mu_{24}^{y} E_{2}-3 \eta_{2}^{2} \mu_{24}^{\prime} E_{2}\right)$

$\nu_{l}^{+}=\nu_{l}^{0+}+\sum_{i=1}^{6} \psi_{l i}^{+} \varepsilon_{i}$,

$\nu_{1}^{0+}=\frac{1}{4}\left(J_{11}^{0}+J_{13}^{0}\right) ; \quad \psi_{1 i}^{+}=\frac{1}{4}\left(\psi_{11 i}+\psi_{13 i}\right)$,

$\nu_{2}^{0+}=\frac{1}{4}\left(J_{12}^{0}+J_{14}^{0}\right) ; \quad \psi_{2 i}^{+}=\frac{1}{4}\left(\psi_{12 i}+\psi_{14 i}\right)$,

$\nu_{3}^{0+}=\frac{1}{4}\left(J_{22}^{0}+J_{24}^{0}\right) ; \quad \psi_{3 i}^{+}=\frac{1}{4}\left(\psi_{22 i}+\psi_{24 i}\right)$.

\section{Thermodynamic Characteristics of GPI in the Electric Field $\boldsymbol{E}_{2}$}

For the calculation of the dielectric, piezoelectric, and elastic characteristics of the GPI, we use the thermodynamic potential per one primitive cell obtained in the two-particle cluster approximation:

$$
\begin{aligned}
& g=\frac{G}{N}=U_{\text {seed }}+H^{0}+\sum_{f=1}^{4} \eta_{f}^{3} \mu_{f}^{\prime} E_{2}- \\
& -2\left(w^{0}+\sum_{i=1}^{6} \delta_{i} \varepsilon_{i}\right)-\frac{1}{2} k_{\mathrm{B}} T \sum_{f=1}^{4} \ln \left(1-\eta_{f}^{2}\right)- \\
& -2 k_{\mathrm{B}} T \ln D+2 k_{\mathrm{B}} T \ln 2-N v \sum_{i=1}^{6} \sigma_{i} \varepsilon_{i} .
\end{aligned}
$$

Minimizing the thermodynamic potential with respect to the strains $\varepsilon_{i}$, we have obtained the following equations for the strains:

$$
\begin{aligned}
& \sigma_{l}=c_{l 1}^{E 0} \varepsilon_{1}+c_{l 2}^{E 0} \varepsilon_{2}+c_{l 3}^{E 0} \varepsilon_{3}+c_{l 5}^{E 0} \varepsilon_{5}-e_{2 l}^{0} E_{2}- \\
& -\frac{2 \delta_{l}}{v}+\frac{2 \delta_{l}}{v D} M_{\varepsilon}-\frac{\psi_{11 l}}{8 v}\left(\eta_{1}^{2}+\eta_{3}^{2}\right)-\frac{\psi_{13 l}}{4 v} \eta_{1} \eta_{3}- \\
& -\frac{\psi_{22 l}}{8 v}\left(\eta_{2}^{2}+\eta_{4}^{2}\right)-\frac{\psi_{24 l}}{4 v} \eta_{2} \eta_{4}-\frac{\psi_{12 l}}{4 v}\left(\eta_{1} \eta_{2}+\eta_{3} \eta_{4}\right)-
\end{aligned}
$$

$-\frac{\psi_{14 l}}{4 v}\left(\eta_{1} \eta_{4}+\eta_{2} \eta_{3}\right) \quad(l=1,2,3,5)$

$\sigma_{4}=c_{44}^{E 0} \varepsilon_{4}+c_{46}^{E 0} \varepsilon_{6}-e_{14}^{0} E_{1}-e_{34}^{0} E_{3}-$

$-\frac{2 \delta_{4}}{v}+\frac{2 \delta_{4}}{v D} M_{\varepsilon}-\frac{\psi_{114}}{8 v}\left(\eta_{1}^{2}+\eta_{3}^{2}\right)-\frac{\psi_{134}}{4 v} \eta_{1} \eta_{3}-$

$-\frac{\psi_{224}}{8 v}\left(\eta_{2}^{2}+\eta_{4}^{2}\right)-\frac{\psi_{244}}{4 v} \eta_{2} \eta_{4}-$

$-\frac{\psi_{124}}{4 v}\left(\eta_{1} \eta_{2}+\eta_{3} \eta_{4}\right)-\frac{\psi_{144}}{4 v}\left(\eta_{1} \eta_{4}+\eta_{2} \eta_{3}\right)$,

$\sigma_{6}=c_{46}^{E 0} \varepsilon_{4}+c_{66}^{E 0} \varepsilon_{6}-e_{16}^{0} E_{1}-e_{36}^{0} E_{3}-$

$-\frac{2 \delta_{6}}{v}+\frac{2 \delta_{6}}{v D} M_{\varepsilon}-\frac{\psi_{116}}{8 v}\left(\eta_{1}^{2}+\eta_{3}^{2}\right)-\frac{\psi_{136}}{4 v} \eta_{1} \eta_{3}-$

$-\frac{\psi_{226}}{8 v}\left(\eta_{2}^{2}+\eta_{4}^{2}\right)-\frac{\psi_{246}}{4 v} \eta_{2} \eta_{4}-$

$-\frac{\psi_{126}}{4 v}\left(\eta_{1} \eta_{2}+\eta_{3} \eta_{4}\right)-\frac{\psi_{146}}{4 v}\left(\eta_{1} \eta_{4}+\eta_{2} \eta_{3}\right)$.

Here,

$M_{\varepsilon}=2 a^{2} \operatorname{ch} n_{3}+2 a^{2} \operatorname{ch} n_{4}+$

$+a \operatorname{ch} n_{5}+a \operatorname{ch} n_{5}+a \operatorname{ch} n_{7}+a \operatorname{ch} n_{8}$.

Differentiating the thermodynamic potential with respect to the fields $E_{i}$, we get the following expressions for the polarizations:

$P_{1}=e_{14}^{0} \varepsilon_{4}+e_{16}^{0} \varepsilon_{6}+\chi_{11}^{\varepsilon 0} E_{1}+$

$+\frac{1}{2 v}\left[\mu_{13}^{x}\left(\eta_{1}-\eta_{3}\right)-\mu_{24}^{x}\left(\eta_{2}-\eta_{4}\right)\right]$,

$P_{2}=e_{21}^{0} \varepsilon_{1}+e_{22}^{0} \varepsilon_{2}+e_{23}^{0} \varepsilon_{3}+e_{25}^{0} \varepsilon_{5}+\chi_{22}^{\varepsilon 0} E_{2}+$

$+\frac{1}{2 v}\left[\mu_{13}^{y}\left(\eta_{1}+\eta_{3}\right)-\mu_{24}^{y}\left(\eta_{2}+\eta_{4}\right)\right]+\frac{1}{2 v} \sum_{f=1}^{4} \eta_{f}^{3} \mu_{f}^{\prime}$,

$P_{3}=e_{34}^{0} \varepsilon_{4}+e_{66}^{0} \varepsilon_{6}+\chi_{33}^{\varepsilon 0} E_{3}+$

$+\frac{1}{2 v}\left[\mu_{13}^{z}\left(\eta_{1}-\eta_{3}\right)+\mu_{24}^{z}\left(\eta_{2}-\eta_{4}\right)\right]$.

The static isothermic dielectric susceptibilities of mechanically clamped crystal GPI are given by

$$
\begin{aligned}
& \chi_{11}^{\varepsilon}=\lim _{E_{1} \rightarrow 0}\left(\frac{\partial P_{1}}{\partial E_{1}}\right)_{\varepsilon_{j}}=\chi_{11}^{\varepsilon 0}+ \\
& +\frac{\beta}{2 v \Delta_{1,3}}\left\{\left(\mu_{13}^{x}\right)^{2}\left[\widetilde{D} \lambda_{24}-\left(\lambda_{13} \lambda_{24}-\lambda^{2}\right) \tilde{\varphi}_{24}^{-}\right]+\right. \\
& +\left(\mu_{24}^{x}\right)^{2}\left[\widetilde{D} \lambda_{13}-\left(\lambda_{13} \lambda_{24}-\lambda^{2}\right) \tilde{\varphi}_{13}^{-}\right]- \\
& \left.-2 \mu_{13}^{x} \mu_{24}^{x}\left[\widetilde{D} \lambda+\left(\lambda_{13} \lambda_{24}-\lambda^{2}\right) \beta \nu_{2}^{-}\right]\right\}
\end{aligned}
$$

ISSN 2071-0194. Ukr. J. Phys. 2018. Vol. 63, No. 4 


$$
\begin{aligned}
& \chi_{22}^{\varepsilon}=\lim _{E_{2} \rightarrow 0}\left(\frac{\partial P_{2}}{\partial E_{2}}\right)_{\varepsilon_{j}}=\chi_{22}^{\varepsilon 0}+ \\
& +\frac{\beta}{2 v \Delta_{2}}\left\{\left(\tilde{\mu}_{13}^{y}\right)^{2}\left[\widetilde{D} \varkappa_{13}-\left(\varkappa_{13} \varkappa_{24}-\varkappa^{2}\right) \tilde{\varphi}_{24}^{+}\right]+\right. \\
& +\left(\tilde{\mu}_{24}^{y}\right)^{2}\left[\widetilde{D} \varkappa_{24}-\left(\varkappa_{13} \varkappa_{24}-\varkappa^{2}\right) \tilde{\varphi}_{13}^{+}\right]- \\
& \left.-2 \tilde{\mu}_{13}^{y} \tilde{\mu}_{24}^{y}\left[\widetilde{D} \varkappa+\left(\varkappa_{13} \varkappa_{24}-\varkappa^{2}\right) \beta \nu_{2}^{+}\right]\right\}, \\
& \chi_{33}^{\varepsilon}=\lim _{E_{3} \rightarrow 0}\left(\frac{\partial P_{3}}{\partial E_{3}}\right)_{\varepsilon_{j}}=\chi_{33}^{\varepsilon 0}+ \\
& +\frac{\beta}{2 v \Delta_{1,3}}\left\{\left(\mu_{13}^{z}\right)^{2}\left[\widetilde{D} \lambda_{24}-\left(\lambda_{13} \lambda_{24}-\lambda^{2}\right) \tilde{\varphi}_{24}^{-}\right]+\right. \\
& +\left(\mu_{24}^{z}\right)^{2}\left[\widetilde{D} \lambda_{13}-\left(\lambda_{13} \lambda_{24}-\lambda^{2}\right) \tilde{\varphi}_{13}^{-}\right]+ \\
& \left.+2 \mu_{13}^{z} \mu_{24}^{z}\left[\widetilde{D} \lambda+\left(\lambda_{13} \lambda_{24}-\lambda^{2}\right) \beta \nu_{2}^{-}\right]\right\}
\end{aligned}
$$

with the notations

$\Delta_{1,3}=\widetilde{D}^{2}-\widetilde{D}\left[\lambda_{24} \tilde{\varphi}_{13}^{-}+\lambda_{13} \tilde{\varphi}_{24}^{-}+2 \lambda \beta \nu_{2}^{-}\right]+$

$+\left(\lambda_{13} \lambda_{24}-\lambda^{2}\right)\left[\tilde{\varphi}_{13}^{-} \tilde{\varphi}_{24}^{-}-\left(\beta \nu_{2}^{-}\right)^{2}\right]$,

$\Delta_{2}=\widetilde{D}^{2}-\widetilde{D}\left[\varkappa_{13} \tilde{\varphi}_{13}^{+}+\varkappa_{24} \tilde{\varphi}_{24}^{+}+2 \varkappa \beta \nu_{2}^{+}\right]+$

$+\left(\varkappa_{13} \varkappa_{24}-\varkappa^{2}\right)\left[\tilde{\varphi}_{13}^{+} \tilde{\varphi}_{24}^{+}-\left(\beta \nu_{2}^{+}\right)^{2}\right]$,

$\varphi_{13}^{ \pm}=\frac{1}{1-\eta_{13}^{2}}+\beta \nu_{1}^{ \pm}, \varphi_{24}^{ \pm}=\frac{1}{1-\eta_{24}^{2}}+\beta \nu_{3}^{ \pm}$,

$\tilde{\varphi}_{13}^{ \pm}=\varphi_{13}^{ \pm}+3 \beta \mu_{13}^{\prime} \eta_{13} E_{2}, \quad \tilde{\varphi}_{24}^{ \pm}=\varphi_{24}^{ \pm}+3 \beta \mu_{24}^{\prime} \eta_{24} E_{2}$,

$\tilde{\mu}_{13}^{y}=\mu_{13}^{y}+3 \mu_{13}^{\prime} \eta_{13}^{2}, \quad \tilde{\mu}_{24}^{y}=\mu_{24}^{y}-3 \mu_{24}^{\prime} \eta_{24}^{2}$,

$\nu_{l}^{-}=\nu_{l}^{0-}+\sum_{i=1}^{6} \psi_{l i}^{-} \varepsilon_{i}$,

$\nu_{1}^{0-}=\frac{1}{4}\left(J_{11}^{0}-J_{13}^{0}\right) ; \quad \psi_{1 i}^{-}=\frac{1}{4}\left(\psi_{11 i}-\psi_{13 i}\right)$,

$\nu_{2}^{0-}=\frac{1}{4}\left(J_{12}^{0}-J_{14}^{0}\right) ; \quad \psi_{2 i}^{-}=\frac{1}{4}\left(\psi_{12 i}-\psi_{14 i}\right)$,

$\nu_{3}^{0-}=\frac{1}{4}\left(J_{22}^{0}-J_{24}^{0}\right) ; \quad \psi_{3 i}^{-}=\frac{1}{4}\left(\psi_{22 i}-\psi_{24 i}\right)$,

$\lambda_{13}=1+a^{2}+2 a \operatorname{ch} y_{13}$,

$\lambda_{24}=1+a^{2}+2 a \operatorname{ch} y_{24}, \quad \lambda=1-a^{2}$,

$\varkappa_{13}=\operatorname{ch}\left(y_{13}+y_{24}\right)+a^{2} \operatorname{ch}\left(y_{13}-y_{24}\right)+$

$+2 a \operatorname{ch} y_{13}-\eta_{13}^{2} \widetilde{D}$,

$\varkappa_{24}=\operatorname{ch}\left(y_{13}+y_{24}\right)+a^{2} \operatorname{ch}\left(y_{13}-y_{24}\right)+$

$+2 a \operatorname{ch} y_{24}-\eta_{24}^{2} \widetilde{D}$,

$\varkappa=\operatorname{ch}\left(y_{13}+y_{24}\right)-a^{2} \operatorname{ch}\left(y_{13}-y_{24}\right)-\eta_{13} \eta_{24} \widetilde{D}$.

ISSN 2071-0194. Ukr. J. Phys. 2018. Vol. 63, No. 4
Differentiating expressions (3.3) with respect to strains $\varepsilon_{i}$ at a constant field, we obtain the expressions for the isothermic coefficients of the piezoelectric stress $e_{2 i}(i=1,2,3,5)$ :

$e_{2 i}=\left(\frac{\partial P_{2}}{\partial \varepsilon_{i}}\right)_{E_{2}}=e_{2 i}^{0}+\frac{\tilde{\mu}_{13}^{y}}{v} \gamma_{13 i}-\frac{\tilde{\mu}_{24}^{y}}{v} \gamma_{24 i}$,

where

$\gamma_{13 i}=\frac{\beta}{\Delta_{2}}\left[\left(\psi_{1 i} \eta_{13}+\psi_{2 i} \eta_{24}\right) \tau_{1}^{\psi}+\right.$

$\left.+\left(\psi_{2 i} \eta_{13}+\psi_{3 i} \eta_{24}\right) \tau_{2}^{\psi}-2 \delta_{i} \tau_{1}^{\delta}\right]$,

$\gamma_{24 i}=\frac{\beta}{\Delta_{2}}\left[\left(\psi_{1 i} \eta_{13}+\psi_{2 i} \eta_{24}\right) \tau_{2}^{\psi}+\right.$

$\left.+\left(\psi_{2 i} \eta_{13}+\psi_{3 i} \eta_{24}\right) \tau_{3}^{\psi}-2 \delta_{i} \tau_{2}^{\delta}\right]$,

$\tau_{1}^{\psi}=\widetilde{D} \varkappa_{13}-\left(\varkappa_{13} \varkappa_{24}-\varkappa^{2}\right) \tilde{\varphi}_{24}^{+}$,

$\tau_{2}^{\psi}=\widetilde{D} \varkappa+\left(\varkappa_{13} \varkappa_{24}-\varkappa^{2}\right) \beta \nu_{2}^{+}$,

$\tau_{3}^{\psi}=\widetilde{D} \varkappa_{24}-\left(\varkappa_{13} \varkappa_{24}-\varkappa^{2}\right) \tilde{\varphi}_{13}^{+}$,

$\tau_{1}^{\delta}=\widetilde{D} \rho_{13}-\left(\varkappa_{24} \rho_{13}-\varkappa \rho_{24}\right) \tilde{\varphi}_{24}^{+}+$

$+\left(\varkappa_{13} \rho_{24}-\varkappa \rho_{13}\right) \beta \nu_{2}^{+}$,

$\tau_{2}^{\delta}=\left[\widetilde{D} \rho_{24}-\left(\varkappa_{13} \rho_{24}-\varkappa \rho_{13}\right) \tilde{\varphi}_{13}^{+}+\right.$

$+\left(\varkappa_{24} \rho_{13}-\varkappa \rho_{24}\right) \beta \nu_{2}^{+}$,

$\rho_{13}=\left[a^{2} \operatorname{sh}\left(y_{13}-y_{24}\right)+a \operatorname{shy} y_{13}\right]-\eta_{13} M$,

$\rho_{24}=\left[-a^{2} \operatorname{sh}\left(y_{13}-y_{24}\right)+a \operatorname{sh} y_{24}\right]-\eta_{24} M$,

$M=a^{2} \operatorname{ch}\left(y_{13}-y_{24}\right)+a \operatorname{ch} y_{13}+a \operatorname{ch} y_{24}+a^{2}$.

The proton contribution to the elastic constants of GPI is found by differentiating (3.2) with respect to strains at a constant field:

$c_{i j}^{E}=\left(\frac{\partial \sigma_{i}}{\partial \varepsilon_{i}}\right)_{E_{2}}=c_{i j}^{E 0}-$

$-\frac{2 \beta}{v \Delta_{2}}\left\{\left(\psi_{1 i} \eta_{13}+\psi_{2 i} \eta_{24}\right)\left(\psi_{1 j} \eta_{13}+\psi_{2 j} \eta_{24}\right) \tau_{1}^{\psi}+\right.$

$+\left(\psi_{2 i} \eta_{13}+\psi_{3 i} \eta_{24}\right)\left(\psi_{2 j} \eta_{13}+\psi_{3 j} \eta_{24}\right) \tau_{3}^{\psi}+$

$+\left[\left(\psi_{1 i} \eta_{13}+\psi_{2 i} \eta_{24}\right)\left(\psi_{2 j} \eta_{13}+\psi_{3 j} \eta_{24}\right)+\right.$

$\left.\left.+\left(\psi_{2 i} \eta_{13}+\psi_{3 i} \eta_{24}\right)\left(\psi_{1 j} \eta_{13}+\psi_{2 j} \eta_{24}\right)\right] \tau_{2}^{\psi}\right\}+$ $+\frac{4 \beta \delta_{i}}{v \Delta_{2}}\left[\left(\psi_{1 j} \eta_{13}+\psi_{2 j} \eta_{24}\right) \tau_{1}^{\delta}+\left(\psi_{2 j} \eta_{13}+\psi_{3 j} \eta_{24}\right) \tau_{2}^{\delta}\right]+$ $+\frac{4 \beta \delta_{j}}{v \Delta_{2}}\left[\left(\psi_{1 i} \eta_{13}+\psi_{2 i} \eta_{24}\right) \tau_{1}^{\delta}+\left(\psi_{2 i} \eta_{13}+\psi_{3 i} \eta_{24}\right) \tau_{2}^{\delta}\right]-$ $-\frac{8 \beta \delta_{i} \delta_{j}}{v \widetilde{D} \Delta_{2}}\left\{\left[\rho_{13} \tilde{\varphi}_{13}^{+}+\rho_{24} \beta \nu_{2}^{+}\right] \tau_{1}^{\delta}+\left[\rho_{24} \tilde{\varphi}_{24}^{+}+\rho_{13} \beta \nu_{2}^{+}\right] \tau_{2}^{\delta}\right\}-$ $-\frac{4 \beta \delta_{i} \delta_{j}}{v \widetilde{D}^{2}}\left\{\left[2 a^{2} \operatorname{ch}\left(y_{13}-y_{24}\right)+\right.\right.$

$\left.\left.+a \operatorname{ch} y_{13}+a \operatorname{ch} y_{24}+2 a^{2}\right] \widetilde{D}-2 M^{2}\right\}$. 
Other dielectric, piezoelectric and elastic characteristics of GPI can be found, by using the expressions established above. In particular, the matrix of isothermal elastic compliance at a constant field $s_{i j}^{E}$, which is reciprocal to the matrix of elastic constants $c_{i j}^{E}$, is as follow:

$\hat{C}^{E}=\left(\begin{array}{cccc}c_{11}^{E} & c_{12}^{E} & c_{13}^{E} & c_{15}^{E} \\ c_{12}^{E} & c_{22}^{E} & c_{23}^{E} & c_{25}^{E} \\ c_{13}^{E} & c_{23}^{E} & c_{33}^{E} & c_{35}^{E} \\ c_{15}^{E} & c_{25}^{E} & c_{35}^{E} & c_{55}^{E}\end{array}\right), \quad \hat{S}^{E}=\left(\hat{C}^{E}\right)^{-1}$,

the isothermic constants of a piezoelectric strain

$d_{2 i}=\sum_{j} s_{i j}^{E} e_{2 j} \quad(i, j=1,2,3,5)$,

the isothermal dielectric susceptibility of a mechanically free crystal

$\chi_{22}^{\sigma}=\chi_{22}^{\varepsilon}+\sum_{i} e_{2 i} d_{2 i}$,

and the molar entropy of the proton subsystem

$$
\begin{aligned}
& S=-\frac{R}{4}\left(\frac{\partial g}{\partial T}\right)_{\eta, \varepsilon_{i}}=\frac{R}{4}\left\{-2 \ln 2+\ln \left(1-\eta_{13}\right)+\right. \\
& +\ln \left(1-\eta_{24}\right)+2 \ln \widetilde{D}-2\left(\beta \nu_{1}^{+} \eta_{13}+\beta \nu_{2}^{+} \eta_{24}\right) \eta_{13}- \\
& \left.-2\left(\beta \nu_{2}^{+} \eta_{13}+\beta \nu_{3}^{+} \eta_{24}\right) \eta_{24}+\frac{4 w}{T \widetilde{D}} M\right\}
\end{aligned}
$$

where $R$ is the gas constant.

The molar heat capacity of the proton subsystem of GPI crystals can be found by the differentiation of entropy (3.11):

$$
\Delta C^{\sigma}=T\left(\frac{\partial S}{\partial T}\right)_{\sigma} .
$$

\section{Comparison of Theoretical \\ Results with Experimental Data}

For the calculation of the temperature and field dependences of the dielectric and piezoelectric characteristics of GPI, we have to determine the values of the model parameters. Methods of determination of the these parameters are described in [7]. Their optimal values are presented below.

The volume of a primitive cell of GPI $v_{H}=0.601 \times$ $\times 10^{-21} \mathrm{~cm}^{3}[12]$.
The parameter of short-range interactions $w^{0} / k_{\mathrm{B}}=$ $=820 \mathrm{~K}$; the deformational potentials $\delta_{i}$, which are coefficients of the linear expansion of the parameter $w$ in strains $\varepsilon_{i}$ are as follow: $\tilde{\delta}_{1}=500 \mathrm{~K}, \tilde{\delta}_{2}=600 \mathrm{~K}$, $\tilde{\delta}_{3}=500 \mathrm{~K}, \tilde{\delta}_{4}=150 \mathrm{~K}, \tilde{\delta}_{5}=100 \mathrm{~K}, \tilde{\delta}_{6}=150 \mathrm{~K}$; $\tilde{\delta}_{i}=\delta_{i} / k_{\mathrm{B}} ;(i=1, \ldots, 6)$;

The parameters of long-range interactions $\tilde{\nu}_{1}^{0+}=$ $=\tilde{\nu}_{2}^{0+}=\tilde{\nu}_{3}^{0+}=2.643 \mathrm{~K}, \tilde{\nu}_{1}^{0-}=\tilde{\nu}_{2}^{0-}=\tilde{\nu}_{3}^{0-}=0.2 \mathrm{~K}$, where $\tilde{\nu}_{f}^{0 \pm}=\nu_{f}^{0 \pm} / k_{\mathrm{B}}(f=1,2,3)$; the deformational potentials $\psi_{f i}^{ \pm}(f=1,2,3 ; i=1, \ldots, 6)$, which are coefficients of the linear expansion of the parameters $\nu_{f}^{0 \pm}$ in strains $\varepsilon_{i}$ are as follow: $\tilde{\psi}_{f 1}^{+}=87.9 \mathrm{~K}$, $\tilde{\psi}_{f 2}^{+}=237.0 \mathrm{~K}, \tilde{\psi}_{f 3}^{+}=103.8 \mathrm{~K}, \tilde{\psi}_{f 4}^{+}=149.1 \mathrm{~K}$, $\tilde{\psi}_{f 5}^{+}=21.3 \mathrm{~K}, \tilde{\psi}_{f 6}^{+}=143.8 \mathrm{~K}, \tilde{\psi}_{f i}^{-}=0 \mathrm{~K}$, where $\tilde{\psi}_{f i}^{ \pm}=\psi_{f i}^{ \pm} / k_{\mathrm{B}}$.

The components of the effective dipole moments are equal to $\mu_{13}^{x}=0.4 \times 10^{-18} \mathrm{esu} \cdot \mathrm{cm} ; \mu_{13}^{y}=4.02 \times$ $\times 10^{-18} \mathrm{esu} \cdot \mathrm{cm} ; \mu_{13}^{z}=4.3 \times 10^{-18} \mathrm{esu} \cdot \mathrm{cm} ; \mu_{24}^{x}=$ $=2.3 \times 10^{-18} \mathrm{esu} \cdot \mathrm{cm} ; \mu_{24}^{y}=3.0 \times 10^{-18} \mathrm{esu} \cdot \mathrm{cm}$; $\mu_{24}^{z}=2.2 \times 10^{-18} \mathrm{esu} \cdot \mathrm{cm}$.

Corrections to the effective dipole moments $\mu_{1}^{\prime}=$ $=\mu_{3}^{\prime}=-0.22 \times 10^{-18} \mathrm{esu} \cdot \mathrm{cm}$ and $\mu_{2}^{\prime}=\mu_{4}^{\prime}=0 \mathrm{esu} \cdot \mathrm{cm}$ are found from the condition of agreement of the saturation polarization with experimental data.

The "seed" coefficients of the piezoelectric stress $e_{i j}^{0}$, "seed" dielectric susceptibilities $\chi_{i j}^{\varepsilon 0}$, and "seed" elastic constants $c_{i j}^{E 0}$ are obtained as follow: $e_{i j}^{0}=0.0 \frac{\mathrm{esu}}{\mathrm{cm}^{2}}$;

$$
\begin{aligned}
& \chi_{11}^{\varepsilon 0}=0.1, \quad \chi_{22}^{\varepsilon 0}=0.403, \quad \chi_{33}^{\varepsilon 0}=0.5, \chi_{31}^{\varepsilon 0}=0.0 \\
& c_{11}^{0 E}=26.91 \times 10^{10} \frac{\mathrm{dyn}}{\mathrm{cm}^{2}}, \quad c_{12}^{E 0}=14.5 \times 10^{10} \frac{\mathrm{dyn}}{\mathrm{cm}^{2}}, \\
& c_{13}^{E 0}=11.64 \times 10^{10} \frac{\mathrm{dyn}}{\mathrm{cm}^{2}}, \quad c_{15}^{E 0}=3.91 \times 10^{10} \frac{\mathrm{dyn}}{\mathrm{cm}^{2}}, \\
& c_{22}^{E 0}=\left(64.99-0.04\left(T-T_{c}\right)\right) \times 10^{10} \frac{\mathrm{dyn}}{\mathrm{cm}^{2}}, \\
& c_{23}^{E 0}=20.38 \times 10^{10} \frac{\mathrm{dyn}}{\mathrm{cm}^{2}}, \quad c_{25}^{E 0}=5.64 \times 10^{10} \frac{\mathrm{dyn}}{\mathrm{cm}^{2}}, \\
& c_{33}^{E 0}=24.41 \times 10^{10} \frac{\mathrm{dyn}}{\mathrm{cm}^{2}}, \quad c_{35}^{E 0}=-2.84 \times 10^{10} \frac{\mathrm{dyn}}{\mathrm{cm}^{2}}, \\
& c_{55}^{E 0}=8.54 \times 10^{10} \frac{\mathrm{dyn}}{\mathrm{cm}^{2}}, \quad c_{44}^{E 0}=15.31 \times 10^{10} \frac{\mathrm{dyn}}{\mathrm{cm}^{2}}, \\
& c_{46}^{E 0}=-1.1 \times 10^{10} \frac{\mathrm{dyn}}{\mathrm{cm}^{2}}, \quad c_{66}^{E 0}=11.88 \times 10^{10} \frac{\mathrm{dyn}}{\mathrm{cm}^{2}} .
\end{aligned}
$$

Now, let us consider the results obtained in this paper for the temperature and field dependences of physical characteristics of a GPI crystal at the different values of the strength of the electric field $E_{2}$. We note that the solid lines in the figures mean the results of calculations on the basis of the theory proposed in the present paper (effective dipole moment $\mu(\eta)$ depends on order parameter), whereas the dashed lines, 


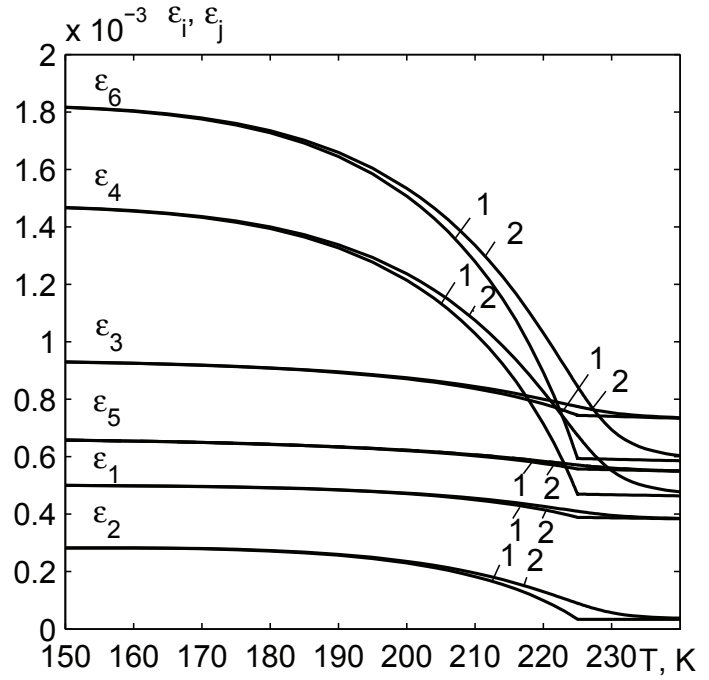

Fig. 2. Temperature dependences of the strains $\varepsilon_{i}$ of a GPI crystal at different values of the electric field $E_{2}(\mathrm{MV} / \mathrm{m}): 1-$ $0.0 ; 2-4.00$

which are given for comparison, mean our results obtained in [7] at the zero field (effective dipole moment is constant, but has different values in the ferro- and paraelectric phases).

The themperature dependences of the strains $\varepsilon_{i}$ of a GPI crystal become smooth near the phase transition temperature $T_{c}=225 \mathrm{~K}$ under the influence of the electric field $E_{2}$ (Fig. 2). At $E_{2}=0$, curves 1 practically coincide with curves obtained in [7]; that is, taking $\mu(\eta)$ into account does not influence practically the strains.

The temperature dependence of the longitudinal polarization $P_{2}(T)$ at $E_{2}=0$ with regard for $\mu(\eta)$ (Fig. 3, curve 1) has larger convexity, in comparison with one obtained in [7] (dashed line).

The curve $P_{2}(T)$ also becomes smooth under the influence of the field $E_{2}$ (Fig. 3, curves 2-5). The polarization $P_{2}$ increases in the whole temperature region with increasing the field strength $E_{2}$. The character of such increase can be seen well on the field dependence of the polarization $P_{2}(E)$ at different temperatures (Fig. 4).

With applying the field $-E_{2}$, the polarization becomes negative, and the curve $P_{2}(E)$ is symmetric relative to such curve at the field $+E_{2}$. At $E_{2}=0$, the curve $P_{2}(E)$ has jump.

In Fig. 5, we present the temperature dependences of the dielectric permittivities of mechanically free

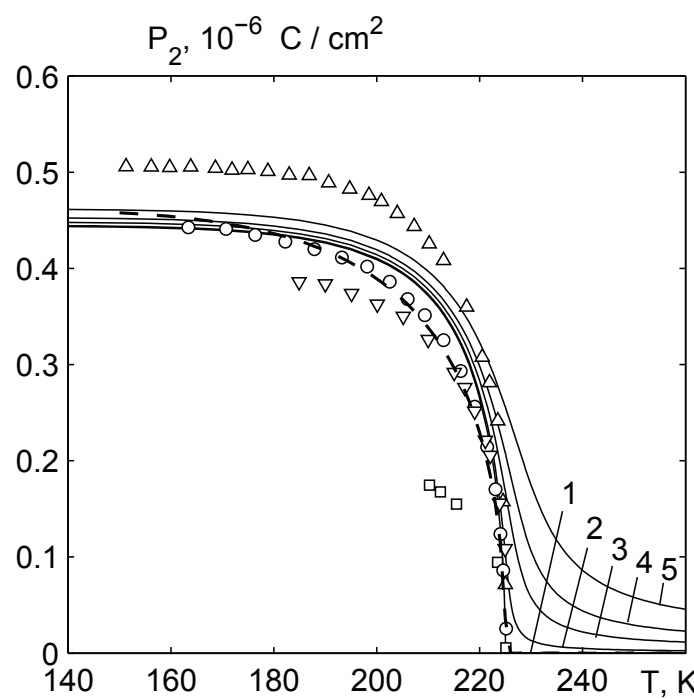

Fig. 3. Temperature dependences of the longitudinal polarization $P_{2}$ of a GPI crystal at different values of the electric field $E_{2}(\mathrm{MV} / \mathrm{m}): 1-0.0 ; \circ[13], \Delta[1], \square[14], \nabla[11] ; 2-0.22$; $3-1.0 ; 4-2.0 ; 5-4.0$. Dashed line is the result obtained in [7]

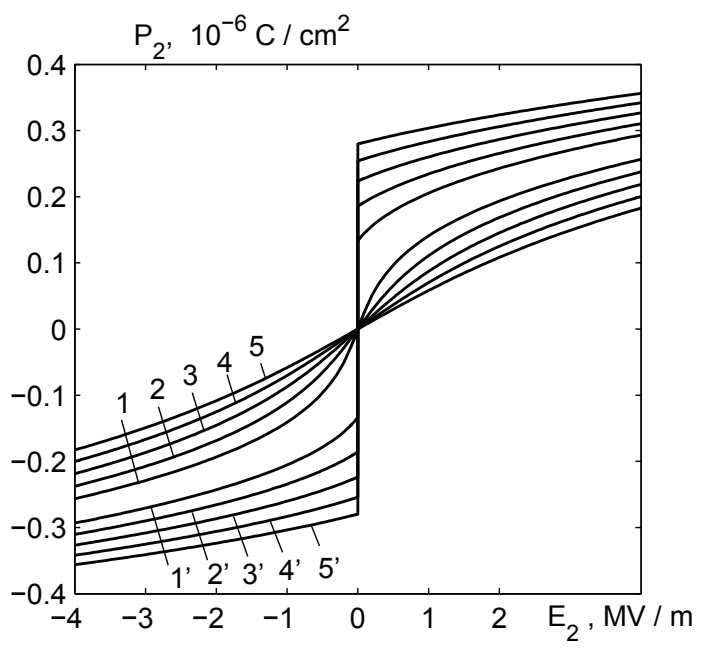

Fig. 4. Dependences of the polarization $P_{2}$ on the field $E_{2}$ at different temperatures $T(\mathrm{~K})$ in the paraelectric phase: 1 226 ; $2-227 ; 3-228 ; 4-229 ; 5-230$; and in the ferroelectric phase: $1^{\prime}-224 ; 2^{\prime}-223 ; 3^{\prime}-222 ; 4^{\prime}-221 ; 5^{\prime}-220$

$\varepsilon_{22}^{\sigma}=1+4 \pi \chi_{22}^{\sigma}$ and clamped $\varepsilon_{22}^{\varepsilon}=1+4 \pi \chi_{22}^{\varepsilon}$ GPI crystals, and the curves $\varepsilon_{22}^{\sigma}(T)$ and $\varepsilon_{22}^{\varepsilon}(T)$ calculated in [7] without regard for the dependence $\mu(\eta)$ (dashed lines).

One can see that the account for $\mu(\eta)$ at the zero electric field leads to increasing the permittivities in 


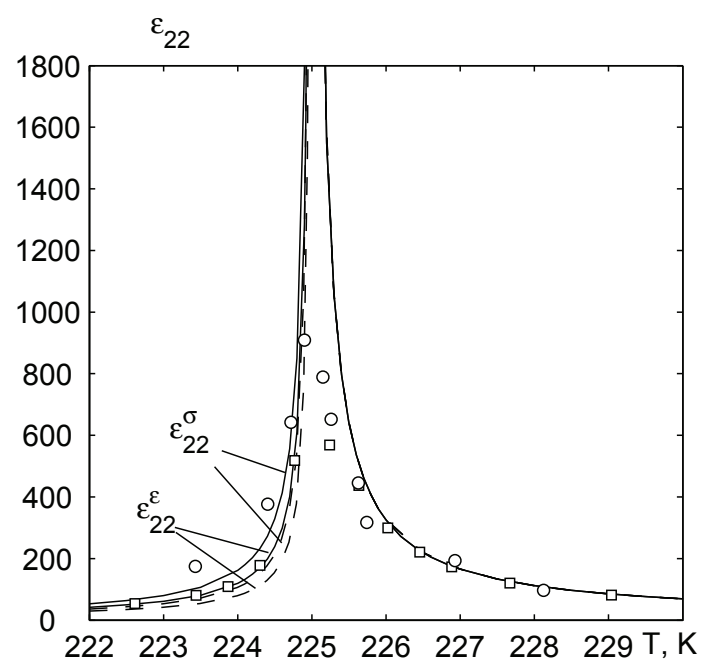

Fig. 5. Temperature dependences of the dielectric permittivities $\varepsilon_{22}^{\sigma}$ and $\varepsilon_{22}^{\varepsilon}$ of a GPI crystal at $E_{2}=0$; ० - [15]; $\square-[14]$. Dashed lines are results obtained in [7]

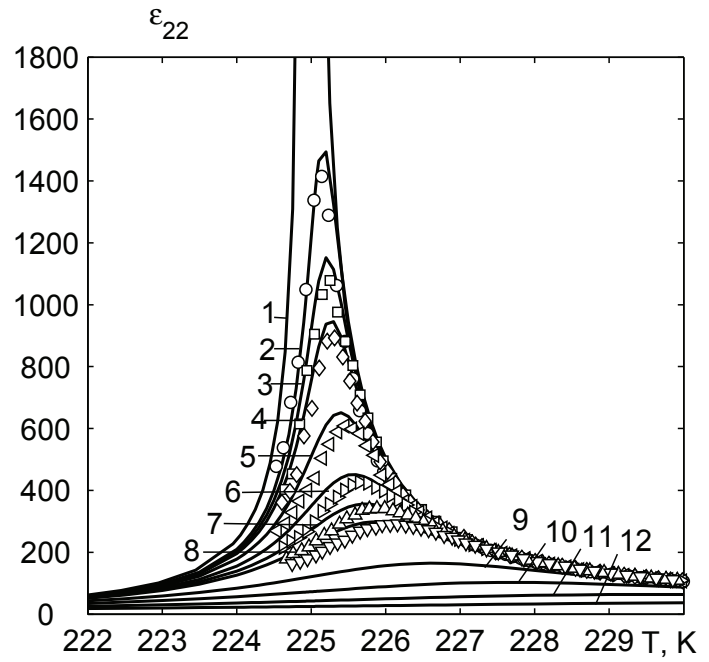

Fig. 6. Temperature dependence of the permittivity $\varepsilon_{22}^{\sigma}$ of a GPI crystal at different values of the electric field $E_{2} \mathrm{MV} / \mathrm{m}$ : $1-0.0 ; 2-0.02 ; \circ ; 3-0.03 ; \square ; 4-0.04 ; \diamond ; 5-0.07 ; \triangle ; 6-$ $0.12 ; \nabla ; 7-0.17 ; \triangleleft ; 8-0.22 ; \triangleright ; 9-0.50 ; 10-1.00 ; 11-2.00$; $12-4.00 ; \triangleright$. Symbols are experimental data taken from [11]

the ferroelectric phase and does not influence them in the paraelectric phase.

The influence of a longitudinal electric field on the temperature dependence of the dielectric permittivity $\varepsilon_{22}^{\sigma}$ of a GPI crystal is presented in Fig. 6 .

With increasing the electric field strength, the maximum on the curve $\varepsilon_{22}^{\sigma}(T)$ becomes smooth, decreases, and shifts to the higher temperatures.

358

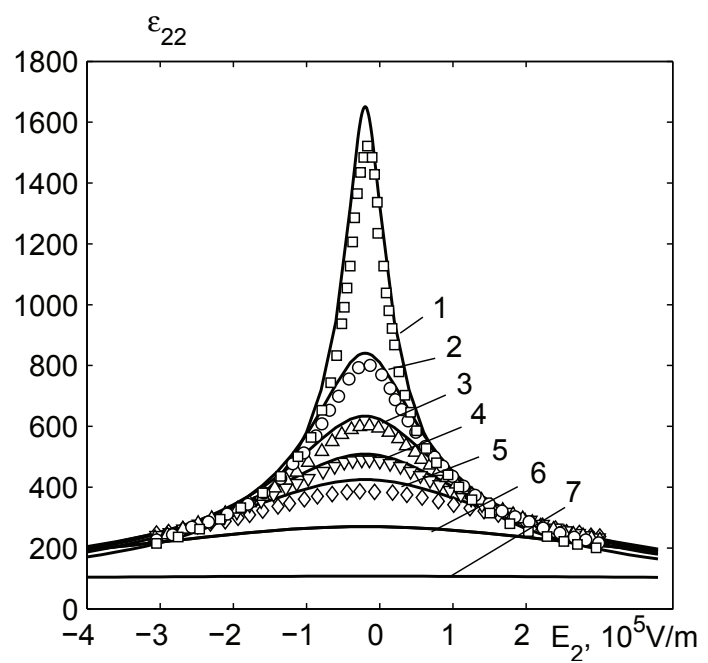

Fig. 7. Dependence of the permittivity $\varepsilon_{22}^{\sigma}$ on the field $E_{2}$ at different temperatures $T(\mathrm{~K}): 1-225.3 ; \square ; 2-225.6$; ०; $3-225.8 ; \Delta ; 4-226.0 ; \nabla ; 5-226.2 \diamond ; 6-227.0 ; 7-230.0$. Symbols are experimental data taken from [11]
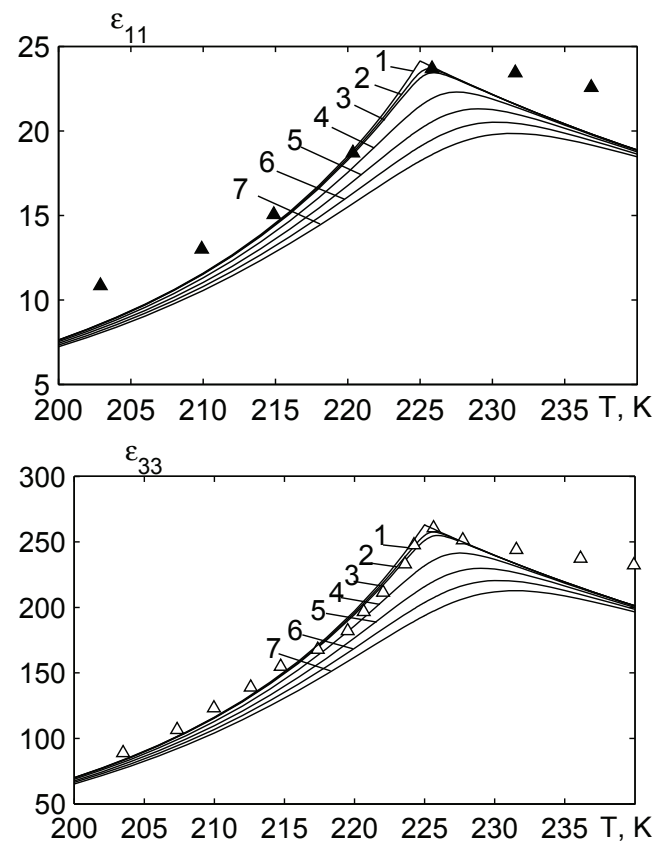

Fig. 8. Temperature dependence of the dielectric permittivity $\varepsilon_{11}$ and $\varepsilon_{33}$ of a GPI crystal at different values of the electric field strength $E_{2} \mathrm{MV} / \mathrm{m}: 1-0.0 ; 2-0.12 ; 3-0.22 ; 4-1.0$; $5-2.0 ; 6-3.0 ; 7-4.0 ;$ symbols $\Delta$ are experimental data of [1]

We note that the curves $\varepsilon_{22}^{\sigma}(T)$, which are calculated at field strengthes $E_{2}=20,30,40,70,120$, 170 , and $220 \mathrm{kV} / \mathrm{m}$, quite well agree with experimental data [11], which are measured at field strengthes

ISSN 2071-0194. Ukr. J. Phys. 2018. Vol. 63, No. 4 

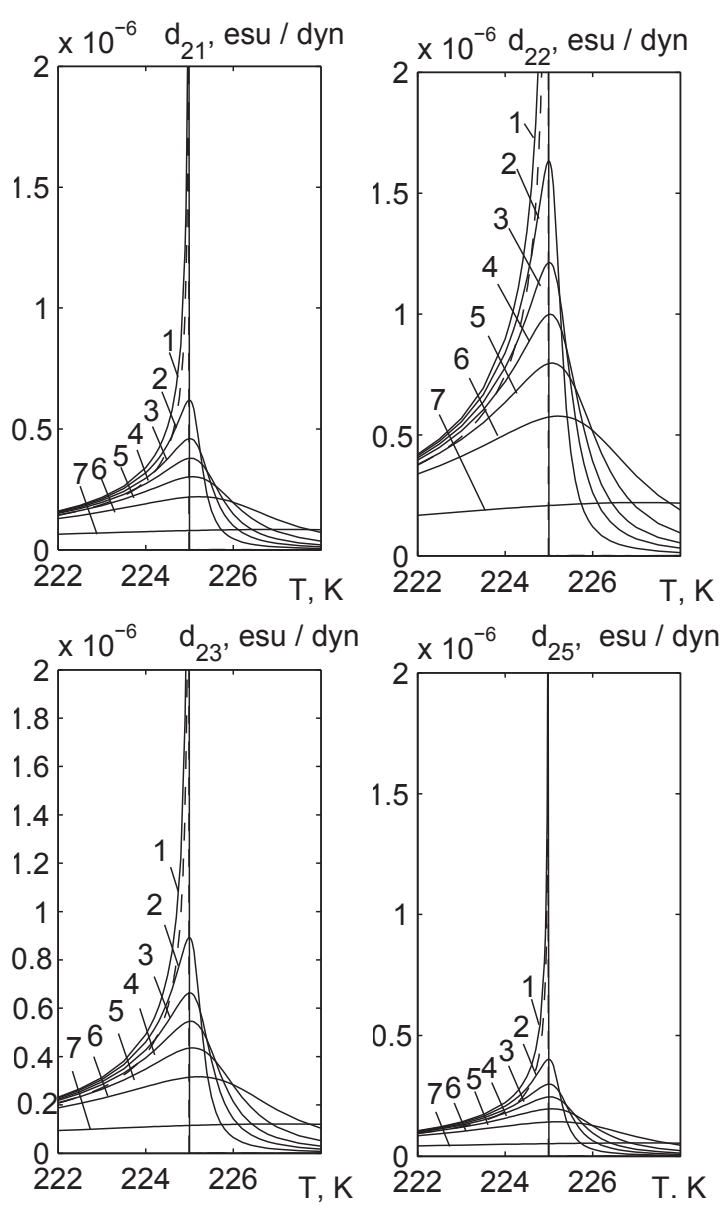

Fig. 9. Temperature dependence of the coefficients of the piezoelectric strains $d_{2 i}$ of a GPI crystal at different values of the electric field strength $E_{2} \mathrm{MV} / \mathrm{m}: 1-0.0 ; 2-0.03 ; 3-0.07$; $4-0.12 ; 5-0.22 ; 6-0.5 ; 7-4.0$. Dashed lines are results calculated in [7] at the zero field

$E_{2}=0,10,20,50,100,150,200 \mathrm{kV} / \mathrm{m}$. This points to the existence of some internal longitudinal electric field $E_{2 i n}=20 \mathrm{kV} / \mathrm{m}$ in the crystal from [11]. The field dependences of $\varepsilon_{22}^{\sigma}(E)$ at different temperatures in the paraelectric phase (Fig. 7) also testify to the existence of an internal field; they have smoothed peaks shifted to $\approx 20 \mathrm{kV} / \mathrm{m}$.

We also note that the experimental data [11] at the zero field do not agree with experimental data of another papers, which we used in [7]. Therefore, for the theoretical description of data [11], we suppose that, in this sample, the parameters $\mu_{13}^{y}=4.28 \times$ $\times 10^{-18} \mathrm{esu} \cdot \mathrm{cm}, \mu_{1}^{\prime}=\mu_{3}^{\prime}=-0.22 \times 10^{-18} \mathrm{esu} \cdot \mathrm{cm}$, but all other parameters are such as obtained in [7].

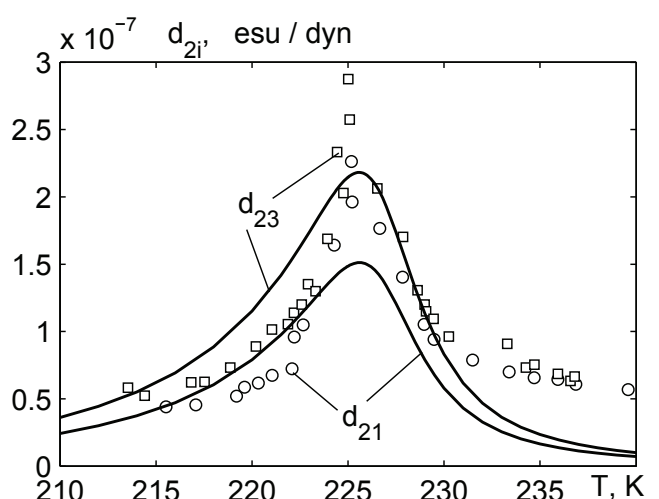

Fig. 10. Temperature dependence of the coefficients of the piezoelectric strains $d_{21}$ and $d_{23}$ of a GPI crystal at the electric field strength $1.2 \mathrm{MV} / \mathrm{m}$. Symbols $\circ, \square$ are experimental data taken from [14]

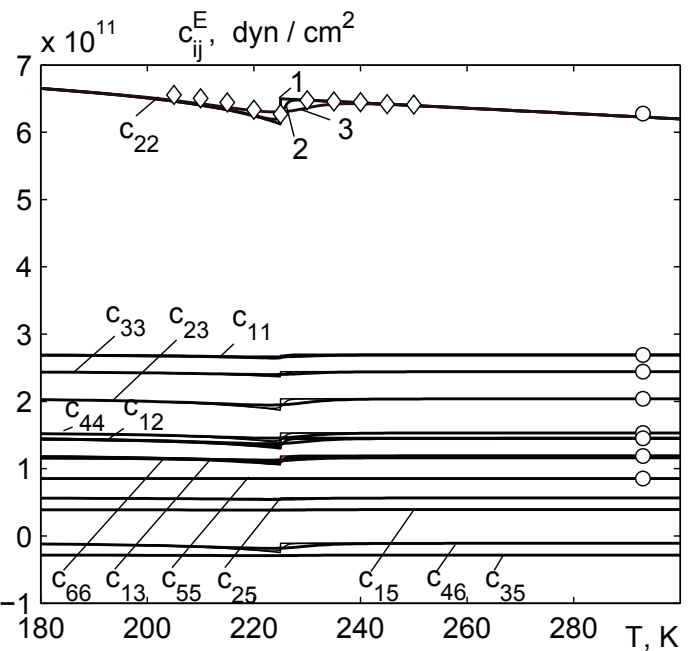

Fig. 11. Temperature dependence of the elastic constants $c_{i j}^{E}$ of a GPI crystal: $c_{11}^{E}-1, c_{12}^{E}-2, c_{13}^{E}-3, c_{22}^{E}-4, c_{23}^{E}-5, c_{33}^{E}-6$, $c_{15}^{E}-7, c_{25}^{E}-8, c_{35}^{E}-9, c_{55}^{E}-10, c_{44}^{E}-11, c_{46}^{E}-12, c_{66}^{E}-13$ at different values of the electric field strength $E_{2} \mathrm{MV} / \mathrm{m}: 1-$ $0.0 ; 2-0.22 ; 3-4.00$. Symbols $\circ$ are experimental data [16]

The temperature dependences of the permittivities $\varepsilon_{11}$ and $\varepsilon_{33}$ of a GPI crystal at the zero field coincide with the curves $\varepsilon_{11}(T)$ and $\varepsilon_{33}(T)$ calculated in [7] without regard for the dependence $\mu(\eta)$. With increasing the field strength, the maxima on the curves $\varepsilon_{11}(T)$ and $\varepsilon_{33}(T)$, as well as in the case of $\varepsilon_{22}(T)$, become smooth, decrease, and shift to higher temperatures (Fig. 8).

From the temperature dependence of the piezoelectric coefficients, $d_{2 i}$ (Fig. 9), one can see that the 


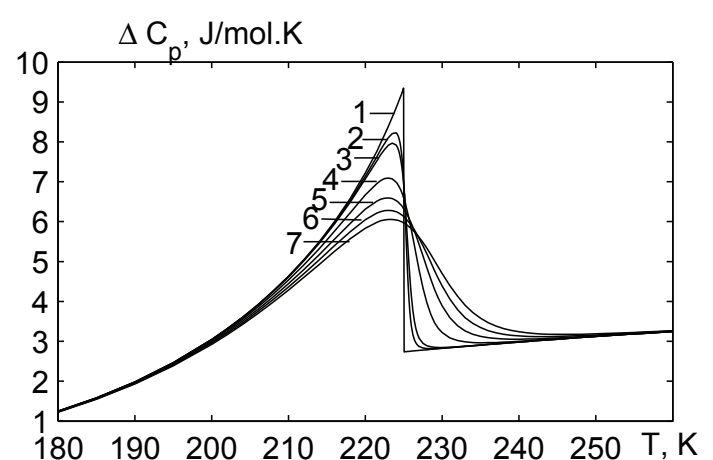

Fig. 12. Temperature dependence of the proton contribution to the molar heat capacity $\triangle C$ of a GPI crystal at different values of $E_{2} \mathrm{MV} / \mathrm{m}: 1-0.0 ; 2-0.12 ; 3-0.22 ; 4-1.0 ; 5-$ $2.0 ; 6-3.0 ; 7-4.0$

account for $\mu(\eta)$ (solid lines) at the zero field increases the coefficients $d_{2 i}$ in comparison with those calculated in [7] without regard for $\mu(\eta)$ (dashed lines). A longitudinal field leads to appearing their nonzero values in the paraelectric phase; and the maxima on the curves $e_{2 i}(T), d_{2 i}(T), h_{2 i}(T)$, and $g_{2 i}(T)$ become smooth, decrease, and shift to higher temperatures.

In [14], the experimental data are obtained for the coefficients of the piezoelectric strains $d_{21}(T)$ and $d_{23}(T)$, which have nonzero values in the paraelectric phase. They may be qualitatively explained by the existence of some internal electric field $\approx 1.2 \mathrm{MV} / \mathrm{m}$ in the sample (Fig. 10).

The account for $\mu(\eta)$ does not influence the temperature dependence of the elastic constants $c_{i j}^{E}$ and the proton contribution to the molar heat capacity $\Delta C$. The role of the electric field $E_{2}$ consists in the smoothering of the curves $c_{i j}^{E}(T)$ (Fig. 11) and $\Delta C(T)$ (Fig. 12).

\section{Conclusions}

On the basis of the proposed model of a deformed GPI crystal, we have calculated its thermodynamic characteristics in the presence of the electric field $E_{2}$. The dependence of the longitudinal component of effective dipole moments on the order parameters $\mu(\eta)$ is taken into account. This allowed us to agree the polarizations in the ferro- and paraelectric phases. The dependence $\mu(\eta)$ leads to a small increase in the polarization in the ferroelectric phase near $T_{c}$, to an increase in the longitudinal permit- tivity in the ferroelectric phase and the piezoelectric coefficients $e_{2 i}$ and $d_{2 i}$, but does not influence the transverse permittivity, longitudinal permittivity in the paraelectric phase, elastic constants, and molar heat capacity.

The calculated temperature and field dependences of thermodynamic characteristics show that an increase in the field $E_{2}$ leads to the smearing of the phase transition, decrease in the dielectric permittivities $\varepsilon_{i i}$ and in the piezoelectric coefficients in the ferroelectric phase for all temperatures. The obtained theoretical dependences have a character of predictions and can stimulate the subsequent experimental investigations.

The authors are indebted to Prof. I. V. Stasyuk for his useful remarks.

1. S. Dacko, Z. Czapla, J. Baran, M. Drozd. Ferroelectricity in Gly $\cdot \mathrm{H}_{3} \mathrm{PO}_{3}$ crystal. Phys. Lett. A 223, 217 (1996).

2. J. Baran, G. Bator, R. Jakubas, M. Sledz. Dielectric dispersion and vibrational studies of a new ferroelectric, glycinium phosphite crystal. J. Phys.: Condens. Matter 8, 10647 (1996)

3. M.-T. Averbuch-Pouchot. Structures of glycinium phosphite and glycylglycinium hosphite. Acta Crystalogr. C 49, 815 (1993).

4. I. Stasyuk, Z. Czapla, S. Dacko, O. Velychko. Proton ordering model of phase transitions in hydrogen bonded ferrielectric type systems: The GPI crystal. Condens. Matter Phys. 6, 483 (2003).

5. I. Stasyuk, Z. Czapla, S. Dacko, O. Velychko. Dielectric anomalies and phase transition in glycinium phosphite crystal under the influence of a transverse electric field. J. Phys.: Condens. Matter 16, 1963 (2004).

6. I. Stasyuk, O. Velychko. Theory of electric field influence on phase transition in glycine phosphite. Ferroelectrics 300, 121 (2004).

7. I.R. Zachek, Ya. Shchur, R.R. Levitskii, A.S. Vdovych. Thermodynamic properties of ferroelectric $\mathrm{NH}_{3} \mathrm{CH}_{2} \mathrm{COOH} \cdot \mathrm{H}_{2} \mathrm{PO}_{3}$ crystal. Physica B 520, 164 (2017).

8. I.R. Zachek, R.R. Levitskii, A.S. Vdovych, I.V. Stasyuk. Influence of electric fields on dielectric properties of GPI ferroelectric. Condens. Matter Phys. 20, 23706 (2017).

9. I.R. Zachek, R.R. Levitskii, A.S. Vdovych. Influence of hydrostatic pressure on thermodynamic characteristics of $\mathrm{NH}_{3} \mathrm{CH}_{2} \mathrm{COOH} \cdot \mathrm{H}_{2} \mathrm{PO}_{3}$ type ferroelectric materials. Condens. Matter Phys. 20, 43707 (2017).

10. I.R. Zachek, R.R. Levitskii, A.S. Vdovych. The influence of uniaxial pressures on thermodynamic properties of the GPI ferroelectric. J. Phys. Study 21, 1704 (2017).

ISSN 2071-0194. Ukr. J. Phys. 2018. Vol. 63, No. 4 
11. R. Tchukvinskyi, R. Cach, Z. Czapla, S. Dacko. Characterization of ferroelectric phase transition in GPI crystal. Phys.stat. sol. (a) 165, 309 (1998).

12. F. Shikanai, M. Yamasaki, M. Komukae, T. Osaka. Structural study of partially deuterated glycinium phosphite in the paraelectric phase. J. Phys. Soc. Jpn. 72, 325 (2003).

13. J. Nayeem, T. Kikuta, N. Nakatani, F. Matsui, S.N. Takeda, K. Hattori, H. Daimon. Ferroelectric phase transition character of glycine phosphite. Ferroelectrics, 332, 13 (2006).

14. M. Wiesner. Piezoelectric properties of GPI crystals. Phys.stat. sol. (b) 238, 68 (2003).

15. J. Nayeem, H. Wakabayashi, T. Kikuta, T. Yamazaki, N. Nakatani. Ferroelectric properties of deuterated glycine phosphite. Ferroelectrics, 269, 153 (2002).

16. A. Deepthy, H.L. Bhat, A.V. Alex, J. Philip. Ultrasonic investigation of elastic properties and a phase transition in ferroelectric glycine phosphite $\mathrm{NH}_{3} \mathrm{CH}_{2} \mathrm{COOH}_{3} \mathrm{PO}_{3}$ single crystals. Phys. Rev. B 62, 8752 (2000).

Received 09.02.18
А.С. Вдович, І.Р. Зачек, Р.Р. Левицъкий

ВПЛИВ ПОЗДОВЖНЬОГО ЕЛЕКТРИЧНОГО ПОЛЯ НА ТЕРМОДИНАМІЧНІ ВЛАСТИВОСТІ СЕГНЕТОЕЛЕКТРИКА $\mathrm{NH}_{3} \mathrm{CH}_{2} \mathrm{COOH} \cdot \mathrm{H}_{2} \mathrm{PO}_{3}$

Р е $з$ ю м е

Використовуючи модифіковану модель сегнетоелектрика фосфіту гліцину шляхом врахування п'єзоелектричного зв'язку $з$ деформаціями $\varepsilon_{i}$ в наближенні двочастинкового кластера розраховано вирази для поляризації, тензора статичної діелектричної проникності кристала, п'єзоелектричних коефіцієнтів і пружних сталих при прикладанні поздовжнього електричного поля $E_{2}$. Проведено аналіз впливу цього поля на термодинамічні характеристики кристала. Враховано залежність ефективних дипольних моментів від параметрів впорядкування. Це дозволило узгодити ефективні дипольні моменти в сегнето- і парафазі та описати розмивання фазового переходу під дією електричного поля. При належному виборі параметрів теорії отримано задовільний кількісний опис наявних експериментальних даних для розрахованих характеристик. 